\title{
Análisis de la velocidad de infiltración del agua en suelos saturados mediante lisímetros de pesada
}

\author{
L. Ávila-Dávila ${ }^{1}$, M. Soler-Méndez ${ }^{2}$, P. Madrona-Sánchez ${ }^{2}$, A. Ruiz-Canales ${ }^{3}$, C.F. Bautista- \\ Capetillo1, J. González-Trinidad1, J.M. Molina-Martínez² \\ 1 Doctorado en Ciencias de la Ingeniería, Universidad Autónoma de Zacatecas, Campus UAZ Siglo XXI, \\ Carretera Zacatecas-Guadalajara Km. 6, Ejido la Escondida, C.P. 98160 Zacatecas, México. \\ laura_14avila@uaz.edu.mx; baucap@uaz.edu.mx; jgonza@uaz.edu.mx \\ 2 Grupo de I+D+i Ingeniería Agromótica y del Mar. Universidad Politécnica de Cartagena, C/ Ángel s/n \\ Ed. ELDI, 30202 Cartagena, España. manuel.ia@agrosolmen.es; josem.molina@upct.es \\ 3 Departamento de Ingeniería, Universidad Miguel Hernández de Elche, Escuela Politécnica Superior de \\ Orihuela, Crtra. De Beniel, km 3,2, 03212 Orihuela (Alicante), España; acanales@umh.es
}

\begin{abstract}
Resumen: La velocidad de infiltración permite estimar la penetración del agua en el suelo, se puede medir como una lámina en función de la profundidad y el tiempo. Existen algunos métodos para medir el comportamiento de la lámina de agua en el suelo. En esta investigación se empleó un lisímetro compacto desarrollado por el grupo de investigación Ingeniería Agromótica y del Mar de la Universidad Politécnica de Cartagena. Se instaló en una finca con suelo de textura franco- limosa, y se registraron los valores minutales del peso del recipiente de cultivo y del depósito de drenaje. Los resultados obtenidos permitieron conocer la evolución de la velocidad de infiltración a lo largo del tiempo. La máxima velocidad de infiltración fue de 0,093 mm/minuto, en el suelo saturado. Con los datos obtenidos en campo se calibró el modelo empírico de Kostiakov, con un error cuadrático medio de 0,00024 . Se concluyó que con el empleo de los nuevos lisímetros de pesada que permiten conocer, en tiempo real, la velocidad de infiltración del agua en suelos saturados, es posible calibrar modelos matemáticos empíricos para su empleo cuando no se dispone de esta nueva tecnología. En el suelo franco-limoso estudiado, el comportamiento fue similar al descrito por Kostiakow, para el cual se obtuvo una ecuación exponencial calibrada.
\end{abstract}

Palabras clave: humedad del suelo, modelo de infiltración, hidrología, balance hídrico

\section{Introducción}

La demanda de agua en la agricultura de regadío es especialmente relevante en países o regiones de clima árido o semiárido, donde se desarrolla bajo un escenario crítico de limitación de agua. Esta situación, hace necesario que se incrementen los esfuerzos para lograr una gestión óptima de los recursos hídricos disponibles para el riego. Se requiere, por tanto, conocer con la mayor exactitud posible el agua que necesita el cultivo para una producción óptima, ya sea en forma de precipitación o de su aplicación desde fuentes superficiales o subterráneas.

La capacidad de retención del agua en el suelo y el movimiento del agua depende de sus características hidrodinámicas. La ecuación de Richards modela el movimiento del agua en el suelo. Se basa en la transferencia de agua en medios porosos considerando la ley de Darcy generalizada y el concepto de potencial de capilaridad [1]. Debido a la complejidad de la estimación de los parámetros de la ecuación de Richard, fueron desarrollados un gran número de modelos empíricos simples de infiltración por [2], [7], [4], entre otros. También, es posible 


\section{CONGRESO IBÉRICO DE AGROINGENIERÍA \\ X CONGRESSO IBÉRICO DE AGROENGENHARIA \\ 3 - 6 septiembre 2019, Huesca - España}

medir la infiltración de manera puntual "IN SITU" por medio de infiltrómetros de cilindro simple o bien doble, los cuales son de fácil operación en una dimensión $[1,5,9]$.

La infiltración es un parámetro que describe la tasa de agua que logra entrar al perfil del suelo. Este parámetro es variable tanto en espacio como en tiempo, debido a que está influenciado por la textura, porosidad, densidad aparente, contenido de materia orgánica, contenido de humedad inicial, compactación del suelo, profundidad del manto freático, y uso del suelo, entre otros [1].

Existen ecuaciones empíricas simples para describir la infiltración en función del tiempo. En algunas ocasiones sus parámetros de ajuste no tienen un significado físico, por lo cual, no representan la infiltración real del suelo y no coinciden con los datos tomados en campo [6,10].

La forma más precisa y fiable de conocer la variación del agua en el suelo es mediante el empleo de lisímetros de pesada. Estos dispositivos permiten realizar experimentos bajo diferentes condiciones (variedad de planta, composición de la tierra, condiciones atmosféricas, horas de riego y duración) y extrapolar el comportamiento a grandes extensiones de terreno, permitiendo de esta manera optimizar los recursos hídricos según sea el caso [3]. Estos dispositivos han sido usados para cuantificar precipitaciones, no solo en forma de lluvia, sino también condensación, y también para determinar la evapotranspiración del cultivo [8].

El objetivo de esta comunicación es mostrar la evolución de la infiltración del agua, considerando las características reales del suelo cuando se desarrolla un cultivo de cebada, a partir de los datos proporcionados por un lisímetro de pesada compacto durante los eventos de lluvia en los que el suelo estuvo saturado, junto con los modelos matemáticos calibrados.

\section{Materiales y métodos}

El experimento se realizó entre febrero y julio de 2017 en una parcela experimental de una hectárea de extensión, llamada "Las Tiesas", perteneciente al Instituto Agronómico Provincial de Albacete (ITAP). El cultivo empleado fue cebada (Hordeum vulgare), variedad "SHAKIRA" de ciclo corto en primavera. El suelo de la parcela experimental es franco-limoso determinado con el triángulo de textura de Marshal, a partir de los porcentajes de las partículas del suelo obtenidos de la prueba de densímetro de Bouyoucos (27,1\% de arena, 51,5\% de limo y 21,4 de arcilla).

Se utilizó el lisímetro de pesada, modelo LP1, diseñado por el grupo de investigación de Ingeniería Agromótica y del Mar de la Universidad Politécnica de Cartagena, cuya precisión de medición es de 0,063 mm de agua. El dispositivo cuenta con un recipiente de cultivo (RC) cuyas dimensiones son $0,96 \mathrm{~m} \times 0,56 \mathrm{~m}$ y 0,35 $\mathrm{m}$ de profundidad en el centro y con depósito de drenaje (DD) utilizado para almacenar y medir el agua, que se filtra a través del volumen de suelo confinado [11].

Por medio de dos sistemas de pesaje, se conocen las variaciones en el contenido de agua del RC y del DD. De esta forma, el lisímetro proporciona una medida directa de las variaciones de peso ocurridas en el RC (provenientes de forma positiva de precipitaciones $(P)$ y riego $(R)$, y de forma negativa del drenaje (D) y de la evapotranspiración (ETc)); así como en el depósito de drenaje (provenientes de forma positiva del drenaje), Figura 1 [12]. La ecuación 1, describe el balance de hídrico, a partir de los datos proporcionados por el lisímetro de pesada:

$$
d \theta / d t=P+R-E T_{C}-E-D
$$

donde $d \theta / d t$ es el cambio volumétrico en el almacenamiento en la humedad del suelo en el perfil del suelo (variación volumétrica del RC) y E es la pérdida dada por la escorrentía [1]. 
X CONGRESO IBÉRICO DE AGROINGENIERÍA

X CONGRESSO IBÉRICO DE AGROENGENHARIA

3 - 6 septiembre 2019, Huesca - España

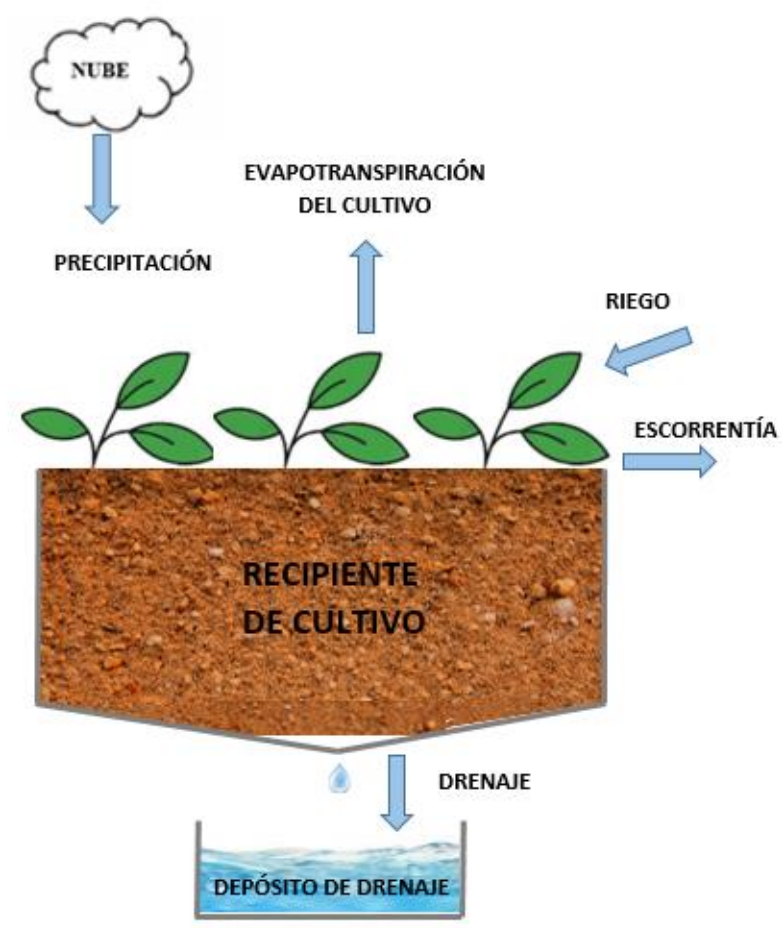

Figura 1. Diagrama de entradas y salidas de agua del recipiente de cultivo y el depósito de drenaje del lisímetro de pesada.

\subsection{Modelo de velocidad de infiltración del agua en suelo saturado con cultivo de cebada}

La metodología que se siguió para obtener el modelo de velocidad de infiltración del agua en el suelo saturado con el cultivo de cebada, consistió en:

1.- Simplificar la ecuación 1, considerando un evento de lluvia, para evitar las pérdidas por evapotranspiración (ETc) y eliminar la entrada de agua del riego (R), quedando la expresión del siguiente modo:

$$
d \theta / d t=P-E-D
$$

2. Descartar el intervalo de tiempo en el que el suelo no alcanzó la saturación; es decir, desde que comenzó el evento de lluvia hasta que el suelo alcanzó la capacidad de campo. Durante ese intervalo, la velocidad de infiltración sigue un modelo de suelo NO saturado, no siendo objeto de estudio en esta comunicación.

3. Determinar la velocidad de infiltración del suelo saturado. Una vez se alcanzó la saturación del suelo, el RC del lisímetro de pesada, empezó a evacuar el agua drenada hacia el DD, siendo medida cada segundo y guardada en el datalogger como promedio minutal. A partir de estos datos, se determinó la velocidad de infiltración del agua en el suelo saturado mediante la siguiente ecuación:

$$
V I=\Delta D D / \Delta t
$$

donde $\Delta D D$ es la variación del peso del depósito de drenaje, en gramos, y $\Delta t$ es la variación del tiempo, en minutos.

Para convertir los gramos medidos por el lisímetro de pesada, a lámina de agua, se empleó la siguiente ecuación: 
X CONGRESO IBÉRICO DE AGROINGENIERÍA

X CONGRESSO IBÉRICO DE AGROENGENHARIA

3 - 6 septiembre 2019, Huesca - España

$$
\text { lámina de agua }=\frac{W}{\rho_{H 2 O} * A_{R C}} * 1000=\frac{W}{A_{R C}}
$$

donde la lámina de agua está en $\mathrm{mm}, W_{R C}$ es el peso del recipiente de cultivo o del depósito de drenaje en $\mathrm{kg}, \rho_{\mathrm{H} 2 O}$ es la densidad del agua $\left(1.000 \mathrm{~kg} / \mathrm{m}^{3}\right)$ y $A_{R C}$ es el área del recipiente de cultivo $\left(0,537 \mathrm{~m}^{2}\right)$.

\subsection{Modelo de Kostiakov}

El modelo que se obtuvo fue comparado con el modelo empírico propuesto por Kostiakov (1932), que establece la siguiente ecuación:

$$
\begin{gathered}
I=a t^{b} \\
V I=d I / d t=a b t^{b-1}
\end{gathered}
$$

donde $I$ es la lámina de agua acumulada que entra al suelo, $t$ es el tiempo de oportunidad de infiltración, a y b son parámetros de ajuste que dependen del suelo y de su condición física y VI es la velocidad de infiltración en $\mathrm{mm} / \mathrm{min}$. Fue elegido este modelo porque la velocidad de infiltración final es cero, que es una de las dos condiciones que establece [1] para el uso de estas ecuaciones.

Los parámetros a y b de Kostiakov se estimaron mediante el método de los mínimos cuadrados a partir de los datos de la lámina infiltrada acumulada (Iacum), obtenida con el lisímetro “IN SITU [13].

\section{Resultados y discusión}

La variación de peso del recipiente de cultivo y del depósito de drenaje monitoreado del 28 de abril hasta el 1 de mayo se presenta en la Figura 2. Los eventos de precipitación de estos cuatro días permitieron llevar al suelo a saturación, lo que ocasionó tres vaciados del depósito de drenaje de los cuales fue posible medir la velocidad de infiltración.

Un suelo saturado permite tener valores fiables y que representan las verdaderas condiciones de campo, en cambio, si es suelo está seco o parcialmente húmedo los valores iniciales de la velocidad de infiltración irán descendiendo con gran rapidez debido a la presión ejercida por el mismo suelo, como en la Figura 3.

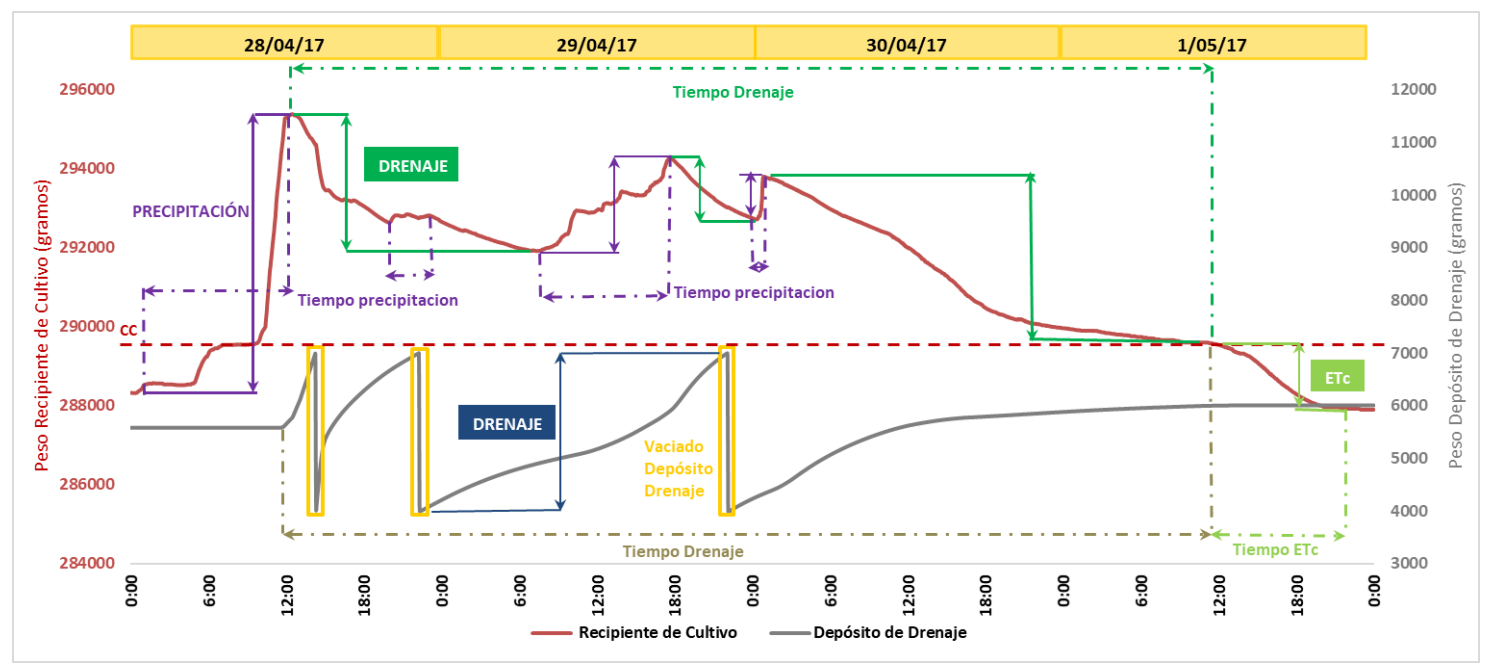

Figura 2. Evolución del peso del recipiente del cultivo y drenaje del 28/04 al 01/05 de 2017, en gramos (g). 


\section{CONGRESO IBÉRICO DE AGROINGENIERÍA \\ X CONGRESSO IBÉRICO DE AGROENGENHARIA \\ 3 - 6 septiembre 2019, Huesca - España}

En la Figura 3, se aprecia la evolución de la velocidad de infiltración en $\mathrm{mm} /$ minuto, función del evento de precipitación que tuvo lugar los días del 28 al 29 de abril. La velocidad máxima de infiltración se alcanzó en el momento en empezó a drenar el DD a las 14:20 horas, la cual generó un valor de $0,093 \mathrm{~mm} /$ minuto, debido a la sorción, a la que determinamos como VI básica, ya que el suelo está saturado [1]. La disminución de la curva conforme va pasando el tiempo se va acercando al cero, esto porque el suelo ya no está siendo capaz de aceptar más agua.

Los momentos de vaciado no afectan el proceso de determinación de la velocidad de infiltración ya que ocurre en un lapso de cuatro minutos, además, la capacidad de saturación ya fue alcanzada por lo que las variaciones del drenado del suelo serán muy pequeñas.

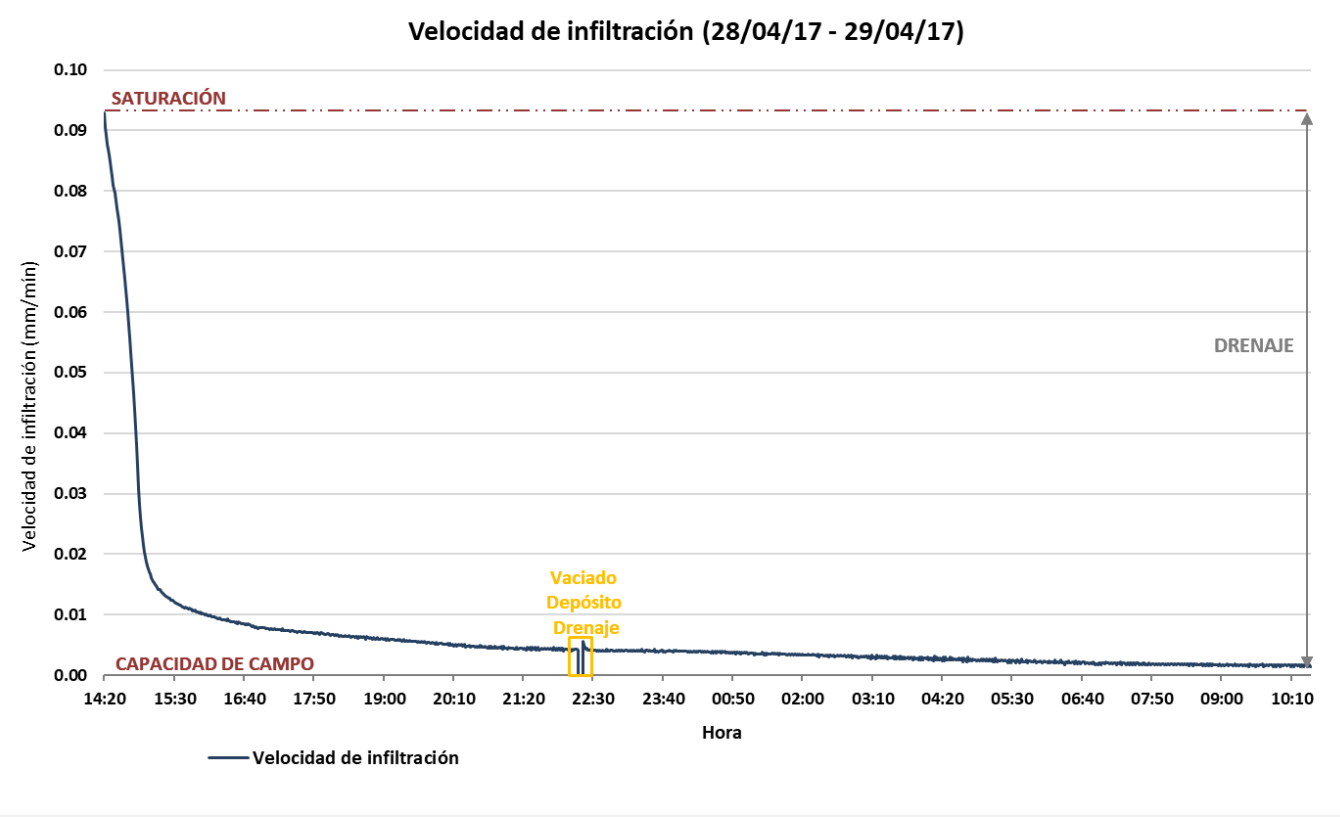

Figura 3. Curva de la velocidad de infiltración del evento de lluvia del 28-29/04/2017

El valor de VI básica obtenido está dentro de los valores estándares para un suelo francolimoso propuesto por [1] y [13] son muy cercanos, $0,08-0,13 \mathrm{~mm} / \mathrm{min}$ y $0,17-0,33 \mathrm{~mm} / \mathrm{min}$, respectivamente. Según [13] la VI básica obtenida puede clasificarse como baja.

Para obtener la ecuación característica de la curva de la Figura 3, se realizó un ajuste de los datos, el mejor fue con una línea de tendencia de potencia presentando un coeficiente de determinación $\left(R^{2}\right)$ de 0,9608 , el modelo obtenido es el siguiente:

$$
V I_{\text {lisimetro }}=0,298 t^{-0,699}
$$

Para la estimación de las constantes de la ecuación de Kostiakov, se obtuvo un a=0,168 y $\mathrm{b}=0,53 \mathrm{El}$ modelo que dio menor error cuadrático medio fue el siguiente:

$$
V I_{\text {Kostiakov }}=0,168(0,53) t^{(0,53-1)}=0,089 t^{-0,47}
$$

A continuación, se muestra las dos curvas de velocidad de infiltración obtenidas con ambos modelos. El caso de la obtenida con el lisímetro de pesada únicamente el primer evento de lluvia, esto porque los eventos consecutivos son muy cercanos a una VI igual a cero (Figura 4). 
X CONGRESO IBÉRICO DE AGROINGENIERÍA

X CONGRESSO IBÉRICO DE AGROENGENHARIA

3 - 6 septiembre 2019, Huesca - España

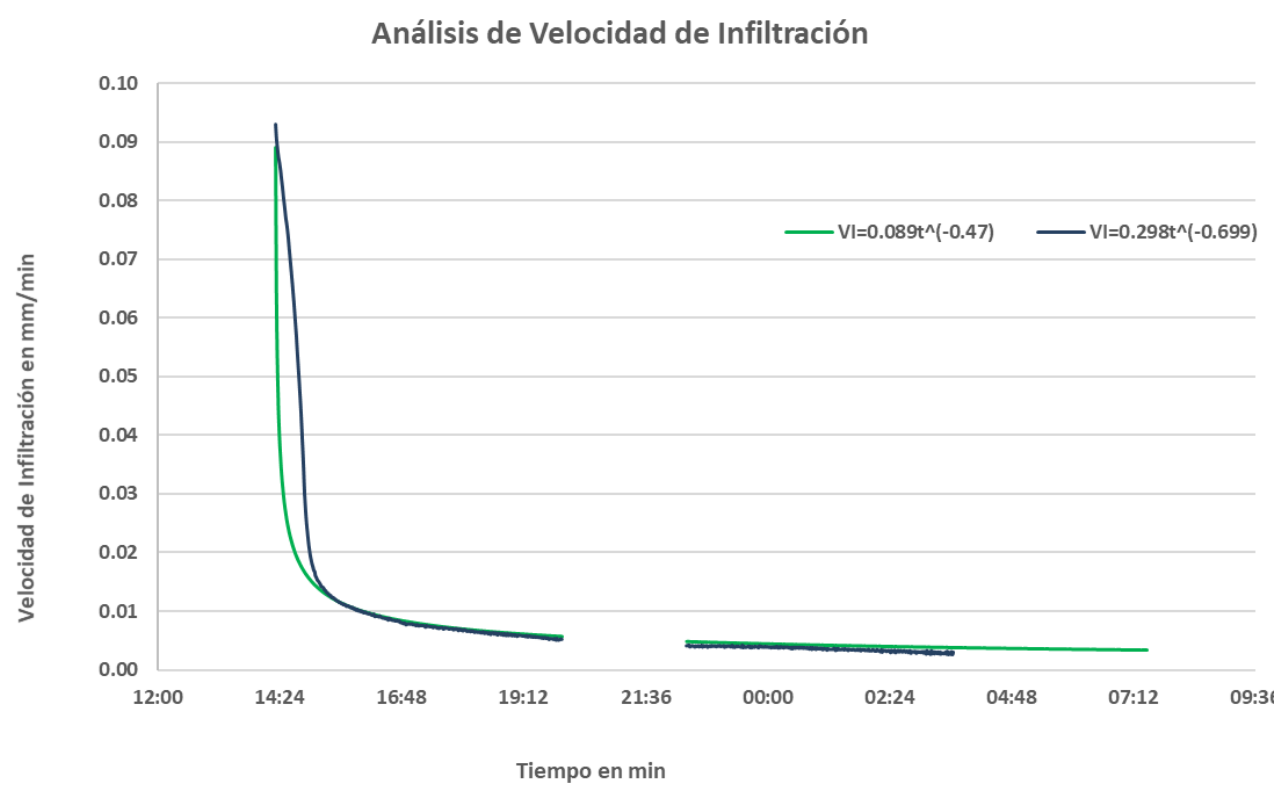

Figura 4. Curvas de los modelos de la velocidad de infiltración del lisímetro y Kostiakov.

El error cuadrático medio entre la velocidad de infiltración obtenida entre lisímetro y la de Kostiakov es de 0,00024, el cual es muy pequeño de acuerdo a los valores recomendados.

Además, fueron validados con los otros eventos de lluvia que hubo del 28 al 30/04/17, los dos modelos obtenidos anteriormente, resultando un error cuadrático de 0,001 con el modelo obtenido con el lisímetro y 0,008 con el modelo de Kostiakov con respecto a los valores medidos.

\section{Conclusiones}

El error cuadrático medio tan cercano a cero entre los dos modelos de Kostiakov y el obtenido con el lisímetro de pesada en el mismo evento de lluvia queda validado, por lo que es posible el empleo de cualquiera de ellos para la estimación de la velocidad de infiltración en este suelo en particular (franco-limoso) cultivado con cebada. Lo cual se comprobó con los errores cuadráticos medios tan pequeños que resultaron en la estimación de las posteriores lluvias al validarlos con los valores reales. Esta investigación seguirá con validaciones en diferentes texturas de suelos, cultivos y con modelos empíricos ya establecidos. Es una investigación a nivel bilateral, entre España y México, con el objetivo de lograr la caracterización del movimiento del agua en el suelo por medio de lisimetría de pesada.

\section{Agradecimientos}

Al Proyecto de Investigación y Desarrollo con referencia IDI-20190146, titulado “Desarrollo e implantación de un equipo de ferticontrol por lisimetría de pesada para uso en agricultura intensiva", en colaboración con la empresa AGROSOLMEN, S.L., cofinanciado por el Fondo Europeo de Desarrollo Regional (FEDER) a través del Programa Operativo Plurirregional de España 2014-2020.

Instituto Técnico Agronómico Provincial de Albacete (ITAP) y al Centro Regional de Estudios del Agua (CREA), por su colaboración para realizar esta investigación.

\section{Referencias}

1. Ali, H. Fundamentals of irrigation and on-farm water management. Springer Science \& Business Media. 2010, volume 1, p. 560. 


\section{CONGRESO IBÉRICO DE AGROINGENIERÍA \\ $X$ CONGRESSO IBÉRICO DE AGROENGENHARIA \\ 3 - 6 septiembre 2019, Huesca - España}

2. Green, W.H. and Ampt, G.A., Studies on soil physics, I. The flow air and wáter through soils. J. Agric. Sci. 1911, 4, 1-24.

3. Jiménez-Buendía, M., Ruiz Peñalver, L., Vera-Repullo, J. A., Intrigliolo-Molina, D. S., Molina-Martínez, J. M. Development and assessment of a network of water meters and rain gauges for determining the water balance. New SCADA monitoring software. Agricultural Water Management. 2014, 151.

4. Kostiakov, A.N. On the dynamics of the coefficient of water percolation in soils and on the necessity for studying it from a dynamic point of view for purposes of amelioration. Trans. Comm. Int. Soil Sci. Soc., A, Moscow, 1932, 17-21.

5. Mahmood, Sajid and Latif, Muhammad. A simple procedure for simulating surge infiltration using first-surge infiltrometer data. Irrigation and Drainage. 2005, 54. 407-416.

6. Mattar, M.A., Alazba, A.A., Zin Elabadin, T.K. Forecasting furrow irrigation infiltration using artificial neural networks. Agricultural Water Management. 2015, 148, 63-71.

7. Philip J. R. “The Theory of Infiltration: 4. Sorptivity and Algebraic Infiltration Equations," Soil Science. 1957, Vol. 84, No. 3, 257-264.

8. Schrader, F., Durner, W., Fank, J., Gebler, S., Pütz, T., Hannes, M., and Wollschläger, U. Estimating precipitation and actual evapotranspiration from precision lysimeter measurements. Procedia Environmental Sciences. 2013, 19, 543-552.

9. Teofilo-Salvador, E., Morales-Reyes, GP. Proposal of the physical model of the multifunctional redesigned double ring infiltrometer (ICCRM). Tecnología y Ciencias del Agua. 2018, 9 (6): 103-131.

10. Yuan, J; Feng, WZ; Jiang, XM; Wang, JL. Saline-alkali migration in soda saline soil based on sub-soiling technology. Desalination and Water Treatment. 2019, 149: 352-362.

11. Nicolás Cuevas J.A., Parras Burgos D., Ruiz Peñalver L., y Molina Martínez J.M. Diseño y modelado gráfico 3D de un lisímetro de pesada de bajo coste. IX Congreso Ibérico de Agroingeniería. 4-6 de septiembre de 2017.

12. Parras Burgos D., Nicolás Cuevas J.A., Ruíz Peñalver L., y Molina Martínez J.M. Evolución del diseño de prototipos de lisímetros de pesada enterrados para cultivos hortícolas. III Symposium Nacional de Ingeniería Hortícola. I Symposium Ibérico de Ingeniería Hortícola. 21-23 de febrero de 2018.

13. Maldonado Rojas T. Manual de Riego Parcelario. Organización de las Naciones Unidas para la Agricultura y la Alimentación, Desarrollo de Tierras y Aguas. Santiago de Chile, febrero del 2001. 